\title{
The role of personality, disability and physical activity in the development of medication-overuse headache: a prospective observational study
}

\author{
Louise S. Mose ${ }^{1,2^{*}}$ (D), Susanne S. Pedersen ${ }^{3,4}$, Birgit Debrabant ${ }^{5}$, Rigmor H. Jensen ${ }^{6}$ and Bibi Gram²
}

\begin{abstract}
Background: Factors associated with development of medication-overuse headache $(\mathrm{MOH})$ in migraine patients are not fully understood, but with respect to prevention, the ability to predict the onset of $\mathrm{MOH}$ is clinically important. The aims were to examine if personality characteristics, disability and physical activity level are associated with the onset of $\mathrm{MOH}$ in a group of migraine patients and explore to which extend these factors combined can predict the onset of $\mathrm{MOH}$.

Methods: The study was a single-center prospective observational study of migraine patients. At inclusion, all patients completed questionnaires evaluating 1) personality (NEO Five-Factor Inventory), 2) disability (Migraine Disability Assessment), and 3) physical activity level (Physical Activity Scale 2.1). Diagnostic codes from patients' electronic health records confirmed if they had developed $\mathrm{MOH}$ during the study period of 20 months. Analyses of associations were performed and to identify which of the variables predict onset $\mathrm{MOH}$, a multivariable least absolute shrinkage and selection operator (LASSO) logistic regression model was fitted to predict presence or absence of $\mathrm{MOH}$.

Results: Out of 131 participants, $12 \%(n=16)$ developed MOH. Migraine disability score (OR=1.02, $95 \%$ Cl: 1.00 to 1.04), intensity of headache ( $\mathrm{OR}=1.49,95 \% \mathrm{Cl}: 1.03$ to 2.15$)$ and headache frequency (OR=1.02, $95 \% \mathrm{Cl}: 1.00$ to 1.04) were associated with the onset of $\mathrm{MOH}$ adjusting for age and gender. To identify which of the variables predict onset $\mathrm{MOH}$, we used a LASSO regression model, and evaluating the predictive performance of the LASSO-mode (containing the predictors MIDAS score, MIDAS-intensity and -frequency, neuroticism score, time with moderate physical activity, educational level, hours of sleep daily and number of contacts to the headache clinic) in terms of area under the curve (AUC) was weak (apparent AUC $=0.62,95 \% \mathrm{Cl}: 0.41-0.82$ ).

Conclusion: Disability, headache intensity and frequency were associated with the onset of $\mathrm{MOH}$ whereas personality and the level of physical activity were not. The multivariable LASSO model based on personality, disability and physical activity is applicable despite moderate study size, however it can be considered as a weak classifier for discriminating between absence and presence of $\mathrm{MOH}$.
\end{abstract}

Keywords: Migraine, Medication-overuse headache, Personality, Disability, Physical activity

\footnotetext{
* Correspondence: Louise.schlosser@rsyd.dk

${ }^{1}$ Department of Neurology, Hospital Southwest Jutland, Esbjerg, Denmark

${ }^{2}$ The Research Unit of Health Science, Hospital of Southwest Jutland, Esbjerg

and Department of Regional Health Research, University of Southern

Denmark, Odense, Denmark

Full list of author information is available at the end of the article
} 


\section{Background}

Migraine is a primary headache disorder characterized by recurring attacks, often described as a one-sided pulsating headache. The mean prevalence of current migraine in adults is around $15 \%, 8 \%$ in men versus $17.6 \%$ in women in Europe [1]. Due to frequent pain-relieving medication intake, migraineurs are predisposed to develop medicationoveruse headache $(\mathrm{MOH})$ [2-5], which is defined as a chronic headache disorder developed as a consequence of regular overuse of acute or symptomatic headache medication [6]. Symptoms of $\mathrm{MOH}$ are often an aggravation and a chronification of the primary headache disorder [7]. Migraine is the primary headache for many $\mathrm{MOH}$ patients, however, not all migraine patients develop $\mathrm{MOH}$. Clearly recognized predictors of developing $\mathrm{MOH}$ are unknown, but potential risk factors such as headache frequency, daily smoking, inactivity, comorbid pain conditions, anxiety and depression have all been discussed in literature [8]. Furthermore, also comorbidity with psychiatric conditions and psychological destress may negatively and significantly modify the outcome for migraine patients [9].

The psychological profile of migraine patients developing $\mathrm{MOH}$ is not fully understood, but the association between migraine and personality has been a topic of interest for many years $[10,11]$. A common, wellestablished approach for describing personality traits is the Five Factor Model of personality [12, 13]. Theoretically, the Five Factor Model approaches personality as a hierarchical system of personality in terms of five basic independent domains: Neuroticism, extroversion, openness to experience, agreeableness, and conscientiousness [14]. Previous studies have demonstrated that a high score on the personality domain neuroticism is correlated with depression [13]. Both migraine $(-\mathrm{MOH})$ and migraine patients $(+\mathrm{MOH})$ have increased risk of developing depression, but migraine patients $(+\mathrm{MOH})$ have a higher prevalence of depression compared to migraine patients $(-\mathrm{MOH})$ [15]. Both the personality domain neuroticism and extraversion have been linked to general health [16] and a high score on neuroticism has been linked to migraine [17-19]. Additional research is needed to clarify the association between migraine and personality characteristics [10]. The Five Factor Model is commonly used to evaluate psychopathology, however, to the best of our knowledge only few studies have investigated personality characteristics of migraine patients using this framework [17, 20].

It is well-documented that headache patients in general experience decreased quality of life and disabilities [21, 22]. $\mathrm{MOH}$ patients frequently experience severe disability as compared to migraine patients without medication overuse [23, 24], but the role of disability in chronification is so far unknown. In the Global Burden of disease (GBD) study from 2016, migraine was estimated to be the main reason for years lived with disability in the age group 15-49, which represented a significant rise from the year before. The explanation was that MOH in GBD 2016 was attributed to the antecedent headache disorder instead of being reported separately $[25,26]$. The 2016 GBD findings further underline the disabling role of migraine in general and in particular $\mathrm{MOH}$ as a sequela. Studies have shown that $\mathrm{MOH}$ patients have higher Migraine Disability Assessment Questionnaire scores (MIDAS) than migraine patients without medication overuse, indicating a higher degree of disability [21, 23, 24, 27]. Still, there is limited research on whether the MIDAS score in migraine patients correlates with the onset of $\mathrm{MOH}$.

In a systematic review, Rhodes \& Smith [28] concluded in correlation between personality characteristics and physical activity that neuroticism is negatively correlated with physical activity, while extraversion and conscientiousness have a positive correlation to physical activity [28]. A recent Danish cross-sectional study found an association between inactivity, daily smoking, obesity, and $\mathrm{MOH}$ [29]. Similarly, a population-based study investigating risk factors for a new onset of $\mathrm{MOH}$ in chronic headache patients found that physical inactivity and smoking were risk factors for developing $\mathrm{MOH}$ [8]. Evidence is lacking on the role of different intensity levels of physical activity in the development of $\mathrm{MOH}$ among migraine patients.

From a clinical point of view, knowledge of risk factors for the onset of $\mathrm{MOH}$ in patients with an established migraine diagnosis is paramount to prevent development of $\mathrm{MOH}$. Factors such as personality, disability and physical activity level may be interesting as possible predictors of $\mathrm{MOH}$, and at the same time, easy to establish and asses through the patients' medical history. Therefore, the aims of this study were firstly to investigate if personality, disability and physical activity level of migraine patients are associated with the development of $\mathrm{MOH}$ in a group of migraine patients who are in active treatment and secondly, to analyze to which extend these factors together can predict the onset of $\mathrm{MOH}$. We hypothesized that personality, disability and level of physical activity varied between migraine patients $(+\mathrm{MOH})$ and migraine patients $(-\mathrm{MOH})$.

\section{Methods}

\section{Design and participants}

The design was a single-center prospective observational study of patients in active treatment recruited from the multidisciplinary Danish Headache Clinic, Hospital of Southwest Jutland in Denmark, between October 2015 and June 2017. Patients were eligible to participate, if they were between 18-65 years of age and had a primary diagnosis of migraine according to the diagnostic criteria from ICHD-III beta [6]. Exclusion criteria were presence of $\mathrm{MOH}$ based on diagnostic criteria of chronic migraine 
and $\mathrm{MOH}$ from ICHD-III beta [6] at inclusion. To minimize any errors and ambiguity in the personality data, we excluded patients with severe comorbid untreated depression or anxiety and patients diagnosed with personality disorders. All patients received standard treatment at the Headache Clinic, which included consultations with a neurologists or headache nurse every three months. As standard, patients were informed about the risk and criteria for $\mathrm{MOH}$ at the initial consultation. In between consultations, patients had the opportunity to contact a headache nurse by phone or mail.

\section{Procedure}

Patients were informed about the study by the neurologists at the consultations in the Headache Clinic. For logistic reasons, it was only possible to include patients two days a week and to extend the recruitment period to 20 months. Number of consultations in the clinic and years diagnosed with migraine varied, however all included patients were seen regularly in the clinic. Patients completed questionnaires regarding personality, extent of disabilities due to migraine, and physical activity level at the inclusion. Throughout the study period, patients' were followed regularly in the Headache clinic and during these consultations patients' self-reported headache diaries together with the physician's examination and assessment formed the basis of a confirmed diagnosis. Information about patients who developed $\mathrm{MOH}$ were obtained from the hospital electronic health records in June 2017 [6].

\section{Ethics, consent and permissions}

This study was part of a larger study that was approved by the Regional Committees on Health Research Ethics for Southern Denmark (ID S-20140114). It was conducted according to the Helsinki Declaration, meaning all patients were informed both orally and in writing prior to giving written informed consent. Permission was obtained from the Danish Data Protection Agency (2008-58-0035).

\section{Measurements}

\section{NEO Five-Factor Inventory}

We assessed personality with the Danish version of NEO Five-Factor Inventory questionnaire (NEO-FFI-3) [30]. The questionnaire consists of 60 items and is a brief version of the original NEO-PI-R [31]. For pragmatic reasons, the short version was chosen as it is less burdensome to patients as compared to the original version with 240 items. The questionnaire is designed as a hierarchical measure with personality seen as five wellestablished domains, which is also referred to as the Five Factor Model of personality. Each domain is assessed by means of 12 questions. The five domains are: (i)
Neuroticism (e.g. the tendency to experience negative emotions, such as anxiety, fear, and frustration); (ii) Extraversion (e.g. the tendency to be outgoing and talkative); (iii) Openness to experience (e.g. the tendency to be creative and imaginative); (iv) Agreeableness (e.g. the tendency to be empathic and altruistic); and (v) Conscientiousness (e.g. efficient, organized, and having selfcontrol). All questions are answered on a five-point Likert scale from "totally disagree to "totally agree". For each domain a $t$-score is calculated as the sum of the 12 items' score ranging from 12-60 [30]. In the current study, the internal consistency of the domains ranged from 0.74-0.90, measured by Cronbach's alpha, which is considered satisfactory.

\section{Migraine Disability Assessment}

To quantify the extent of disability, the MIDAS questionnaire was used, which is one of the most frequently used measures to assess disability in migraine patients [32-34]. MIDAS consists of 5 items that captures information on disability on a four-point score. The grade of disability is scored as the sum of days with headache during the previous three months that prevented patients from or reduced productivity by at least $50 \%$ with respect to work/school, housework, and social/leisure activities. Furthermore, MIDAS consists of two additional questions on number of days with headache during the previous three months and intensity of headache measured on a numeric rating scale ranging from $0-10$ where 0 is "no pain" and 10 is "worst imaginable pain".

\section{Physical Activity Scale}

To measure physical activity level, the questionnaire Physical Activity Scale 2.1 (PAS 2.1) was used [35]. In PAS 2.1, the patients were asked to specify number of hours and minutes in an average 24-hour day spent on physical activity categorized as i) sleeping, ii) work related sitting/standing/walking and heavy physical work, iii) transportation to or from work (walking/cycling to work), and iv) sedentary leisure time activities (e.g. TV-viewing). Additionally, PAS 2.1 provided estimates on hours and minutes on a weekly basis spent on physical activity at three different intensity levels: 'Light', 'moderate' and 'vigorous' physical activity.

\section{Statistical analyses}

The outcome of interest in this study was whether patients developed $\mathrm{MOH}$ during the study period or not. Baseline demographic characteristics comparing the two groups migraine $(+\mathrm{MOH})$ and migraine $(-\mathrm{MOH})$ were calculated using the chi-square test for larger samples and Fisher's exact for samples less than five categorical data and Mann-Whitney $U$ test for data with skewed distribution and unpaired t-test for data following 
normal distribution. For hypothesis testing we used twotailed test. $P$-values of $<0.05$ were considered statistically significant.

\section{Associations between development of $\mathrm{MOH}$, personality, disability and physical activity}

Using logistic regression, we investigated whether $\mathrm{MOH}$ onset was associated with personality characteristics, disabilities or physical activity level. Each of the following variables were tested in a separate regression model: Unemployment, neuroticism, extraversion, openness, agreeableness, conscientiousness, MIDAS score, MIDAS-intensity and MIDAS-frequency, and physical activity level divided into hours weekly on light, moderate or vigorous activity. All regression models were adjusted for age and gender to avoid confounding effects on both personality score and development of $\mathrm{MOH}[4,30,36-38]$.

\section{Predicting presence or absence of $\mathrm{MOH}$}

To investigate the ability of our variables to jointly predict onset of $\mathrm{MOH}$, we considered a multivariable prediction model obtained by least absolute shrinkage and selection operator (LASSO) regression. This penalized regression method allows for the integration of a large number of possible correlated predictors into one model and to select amongst these despite a small sample size. The following predictors were included: age, gender, civil status, educational level, primary diagnosis, contacts to the headache clinic, the five NEO-FFI-3 domains as separate variables, disability using MIDAS score, intensity and frequency, measurements from PAS 2.1 on times spent for sleeping, sedentary leisure time activities and times for light activities, moderate activities or vigorous activities and a binary variable indicating if patients were unemployed. Remaining variables from the PAS 2.1 assessing different activity levels during work times were not included, since they were not available for patients without employment. Educational level was included both as continuous and as categorical variable. We only used data from patients without missing data in any of the included covariates. Variable standardization prior to fitting was applied, but reported Odds ratios (OR) are returned on the original scale. Due to the LASSO penalty, ORs are biased towards one for the benefit of improved predictions. The tuning parameter controlling the strength of the penalty was chosen to maximize the goodness-of-fit in an 8-fold cross validation. Folds were chosen randomly, but each fold contained two $(+\mathrm{MOH})$ patients. As goodness-of-fit-measure we used the area under the ROC curve (AUC). After the tuning parameter had been determined, we calculated the AUC in the complete dataset together with its $95 \%$ confidence interval. Because the AUC value of our prediction model for new samples from the same population is expected to be below the calculated apparent AUC (especially because of the previous model selection incorporated in the LASSO approach), we applied bootstrap resampling as described in Steyerberg [39] to calculate an optimismcorrected AUC-value. We used 500 bootstrap samples, but discarded those for which the LASSO logistic regression failed to converge. At the same time and using the same approach, we calculated bootstrap based corrections for the lower and upper bound of the corresponding confidence interval.

Statistical analyses were performed with StatalC14 (StataCorp LP, College Station, Texas). We used the statistics software $\mathrm{R}$ (version 3.3.2) together with the packages glmnet version 2.0-10 [40], ROCR version 1.0-7 [41], pROC version 1.10.0 [42] and caret version 6.0-76 [43] to carry out the LASSO logistic regression model, calculate the AUC and its confidence intervals and to plot the ROC curve.

\section{Results}

A total of 156 patients were informed about the study and 131 accepted to participate. Two patients did not want to participate, as they felt uncomfortable about answering the questionnaires, while 23 patients failed to return the questionnaires. Of the 131 included patients, 119 (91\%) received prophylactic treatment for migraine at inclusion and patients had a mean [range] follow-up time of 361[18-631] days. There were no statistically significant differences between non-responders and included patients regarding age, gender and primary headache diagnoses (all $p$-values $>0.05$ ).

\section{Clinical characteristics}

Sixteen migraine patients (12\%) developed $\mathrm{MOH}$, while $88 \%(n=115)$ were still migraine patients without $\mathrm{MOH}$ at the end of the study period. The majority of migraine patients $(-\mathrm{MOH})$ were women $87 \%(n=100)$ with a mean (SD) age of 39.2 (13) years. The distributions of primary headache diagnosis in the migraine $(-\mathrm{MOH})$ group were $38 \%(n=44)$ had tension-type headache (TTH) as comorbidity, while $62 \%(n=71)$ had only migraine. Also in the migraine $(+\mathrm{MOH})$ group, women were predominant by $94 \%(n=15)$ with a mean (SD) age of $37.3(13)$. In this group migraine was primary headache diagnosis for $50 \%$ $(n=8)$ while $50 \%(n=8)$ had comorbidity migraine and TTH. The migraine $(+\mathrm{MOH})$ group had a significantly higher numbers of contacts to the Headache Clinic during the study period; median (IQR) contacts 8.5 (4 to $10)$ as compared to the migraine $(-\mathrm{MOH})$ group with median (IQR) contacts 6 (3 to 7) $(p=0.028)$. No other statistically significant differences in demographic and headache characteristics were observed between the groups. Characteristics are summarized in Table 1. 
Table 1 Patients' demographic and headache characteristics

\begin{tabular}{|c|c|c|c|c|}
\hline & Migraine $(-\mathrm{MOH})(n=115)$ & Migraine $(+\mathrm{MOH})(n=16)$ & All participants $(n=131)$ & $P$-value \\
\hline Age (years)(mean $\pm S D)$ & $39.2 \pm 13$ & $37.3 \pm 13$ & $39.0 \pm 13$ & NS \\
\hline \multicolumn{5}{|l|}{ Sex n (\%) } \\
\hline Female & $100(87)$ & $15(94)$ & $115(88)$ & \multirow[t]{2}{*}{ NS } \\
\hline Male & $15(13)$ & $1(6)$ & $16(12)$ & \\
\hline \multicolumn{5}{|l|}{ Civil status n (\%) } \\
\hline Single & $25(22)$ & $3(19)$ & $28(21)$ & \multirow[t]{2}{*}{ NS } \\
\hline Cohabiting & $90(78)$ & $13(81)$ & $103(79)$ & \\
\hline \multicolumn{5}{|l|}{ Educational level n (\%) } \\
\hline Primary/secondary school & $16(14)$ & $5(31)$ & $21(16)$ & \multirow[t]{3}{*}{ NS } \\
\hline Vocational/High school & $55(48)$ & $7(44)$ & $62(47)$ & \\
\hline Bachelor or higher degree & $44(38)$ & $4(25)$ & $48(37)$ & \\
\hline \multicolumn{5}{|l|}{ Working status n (\%) } \\
\hline Employed/student & $91(79)$ & $13(81)$ & $104(82)$ & \multirow[t]{2}{*}{ NS } \\
\hline Unemployed/sickness benefits/social welfare & $24(21)$ & $3(19)$ & $27(18)$ & \\
\hline Sleep (hours daily) (mean \pm SD) & $8 \pm 1$ & $8 \pm 1$ & $8 \pm 1$ & NS \\
\hline \multicolumn{5}{|l|}{ Primary diagnosis $\mathrm{n}(\%)$} \\
\hline Migraine & $71(62)$ & $8(50)$ & $79(60)$ & \multirow[t]{2}{*}{ NS } \\
\hline Migraine + Tension Type Headache & $44(38)$ & $8(50)$ & $52(40)$ & \\
\hline Contacts headache clinic median [IQR] & $6[3-7]$ & $8.5[4-10]$ & $5[3-8]$ & 0.028 \\
\hline
\end{tabular}

Differences between groups on normal distributed data were tested using unpaired t-test and Chi-square test for samples $>5$ and Fisher's exact for samples $<5$. Data are presented as mean \pm standard deviation (SD) or as numbers with percentages (\%) in brackets. For skewed data Wilcoxon Mann-Whitney test were used and data were presented as median and interquartile range $[\mathrm{IQR}]$ in brackets. $P$-values $<0.05$ were considered as statistically significant for all tests.

Comparison of personality characteristics between groups showed no statistical differences. Migraine $(+\mathrm{MOH})$ had significantly higher headache intensity median (IQR) $7(6.5-8)$ as compared to the migraine $(-\mathrm{MOH})$ group with median (IQR) of $6(5-7),(p=0.041)$. Headache frequency for the previous three months, were also significantly higher among the migraine $(+\mathrm{MOH})$ group with median (IQR) of 46 (28.5-87.5), compared to migraine $(-\mathrm{MOH})$ with median (IQR) of 30 (15-54), $(p=0.017)$ (Table 2). Overall, neither the migraineurs $(-\mathrm{MOH})$ nor the migraineurs $(+\mathrm{MOH})$ were physically active as they spent only few hours weekly on light physical activity, even fewer hours at moderate physical activity and almost no time on vigorous physical activity. No statistical significant differences were found between the groups regarding level of physical activity (Table 2).

\section{Associations between development of $\mathrm{MOH}$, personality, disability and physical activity}

When adjusting for age and gender in the logistic regressions analysis for the onset of $\mathrm{MOH}$, no significant differences in odds were found with respect to unemployment (OR=0.967, 95\% CI: 0.24 to 3.77 ). The personality domains neuroticism $(\mathrm{OR}=1.06,95 \% \mathrm{CI}: 0.99$ to 1.13$)$, extraversion ( $\mathrm{OR}=0.96,95 \% \mathrm{CI}: 0.88$ to 1.04$)$, openness (OR=0.99, $95 \% \mathrm{CI}$ : 0.91 to 1.08$)$, agreeableness
$(\mathrm{OR}=1.00,95 \% \mathrm{CI}: 0.93$ to 1.08$)$ and conscientiousness (OR=0.95, 95 \% CI: 0.87 to 1.03 ) were not associated with onset of $\mathrm{MOH}$ in migraine patients.

Analyses on the relationship between MIDAS score and $\mathrm{MOH}$ demonstrated significant association between MIDAS score (OR=1.02, $95 \% \mathrm{CI}: 1.00$ to 1.04$)$ and intensity of headache (OR=1.49, $95 \% \mathrm{CI}: 1.03$ to 2.15 ) and between MIIDAS score and headache frequency (OR=1.02, 95 \% CI: 1.00 to 1.04$)$.

The three variables describing levels of physical activity were not significantly associated with onset of $\mathrm{MOH}$; light activity $(\mathrm{OR}=1.00,95 \% \mathrm{CI}: 0.93$ to 1.08$)$, moderate activity $(\mathrm{OR}=0.87,95 \% \mathrm{CI}: 0.70$ to 1.07$)$ and vigorous activity (OR=0.91, $95 \% \mathrm{CI}: 0.66$ to 1.26$)$. All association analyses using logistic regression are summarized in Table 3.

\section{Predicting presence or absence of $\mathrm{MOH}$}

Two patients out of 131 were excluded from this analysis due to missing values. Table 4 illustrates our multivariable prediction model and shows the covariates selected by the multivariable LASSO logistic regression together with the estimated ORs.

The predicted odds for developing $\mathrm{MOH}$ increased by $21 \%(\mathrm{OR}=1.210)$ by each unit of headache frequency reported. The predicted odds increased by $0.2 \%(\mathrm{OR}=1.002)$ 
Table 2 Comparison of personality, disability and physical activity levels between migraine $(-\mathrm{MOH})$ and migraine $(+\mathrm{MOH})$. All data are presented as medians [interquartile ranges]

\begin{tabular}{|c|c|c|c|c|}
\hline & Migraine $(-\mathrm{MOH})(n=115)$ & Migraine $(+\mathrm{MOH})(n=16)$ & All participants $(n=131)$ & $P$-value \\
\hline Neuroticism (12-60) & $35[28-42]$ & $38.5[31-46.5]$ & $35[28-42]$ & NS \\
\hline Extraversion (12-60) & 39 [34-44] & $39[30.5-41]$ & $39[33-44]$ & NS \\
\hline Openness (12-60) & 37 [33-42] & $34.5[34-43]$ & $37[33-42]$ & NS \\
\hline Agreeableness (12-60) & $44[39-48]$ & $44.5[39-48.5]$ & $44[39-48]$ & NS \\
\hline Conscientiousness (12-60) & $47[43-51]$ & $46.5[39.5-50]$ & $47[42-51]$ & NS \\
\hline MIDAS score $(0-270)$ & $32[16-57]$ & $42[28-94.5]$ & $33[17-58]$ & NS \\
\hline MIDAS-frequency (days last 3 months) & $30[15-54]$ & $46[28.5-87.5]$ & 30 [18- 63] & 0.017 \\
\hline MIDAS-intensity (NRS 0-10) & $6[5-7]$ & $7[6.5-8]$ & $7[5-7]$ & 0.041 \\
\hline Light physical activity (hours/week) & $5.25[3-10]$ & 7 [4-13] & $6[3-10]$ & NS \\
\hline Moderate physical activity(hours/week) & $2.5[1-5]$ & $2[0.5-4]$ & $2.5[0.5-5]$ & NS \\
\hline Vigorous physical activity (hours/ week) & $0[0-2]$ & $0[0-1.5]$ & $0[0-2]$ & NS \\
\hline
\end{tabular}

Differences between groups were tested using Wilcoxon Mann-Whitney test. $P$-values< 0.05 were considered as statistically significant for all tests. NEO-FFI-3: NEO Five-Factor Inventory. MIDAS: Migraine disability assessment questionnaire. PAS 2.1: Physical Activity Scale questionnaire

for each additional MIDAS score point, and $0.6 \%$ $(\mathrm{OR}=0.006)$ for each unit of intensity of headache reported.

The predicted odds for experiencing $\mathrm{MOH}$ estimated by the LASSO model were $3.2 \%$ higher $(\mathrm{OR}=1.032)$ for each additional hour of sleep, and decreased by $1.7 \%$ $(\mathrm{OR}=0.983)$ for each hour spent on moderate physical activity. Regarding personality domains, an additional unit in the neuroticism score increased the odds by $1.7 \%$ $(\mathrm{OR}=1.017)$. Each additional level of education decreased the odds by $20.6 \%(\mathrm{OR}=0.794)$ and each additional contact to the Headache clinic increased the odds by $1 \%$ $(\mathrm{OR}=1.010)$. Remaining covariates were not part of the selected model.

Evaluating the predictive performance of the LASSOmodel, we obtained the ROC curve, shown in Fig. 1

Table 3 Associations between development of $\mathrm{MOH}$ and personality, disability and physical activity level

\begin{tabular}{llll}
\hline Covariates & OR & $95 \% \mathrm{Cl}$ & $P$-value \\
\hline Unemployment $(n=131)$ & 0.97 & $0.24-3.77$ & 0.961 \\
Light physical activity (hours/week) $(n=130)$ & 1.00 & $0.93-1.08$ & 0.947 \\
Moderate physical activity (hours/week) $(n=130)$ & 0.87 & $0.70-1.07$ & 0.193 \\
Vigorous physical activity (hours/week) $(n=131)$ & 0.91 & $0.66-1.26$ & 0.588 \\
MIDAS score (0-270)( $(n=131)$ & 1.02 & $1.00-1.04$ & 0.032 \\
MIDAS-intensity (NRS 0-10) ( $n=131)$ & 1.49 & $1.03-2.15$ & 0.034 \\
MIDAS-frequency (days last 3 months) $(n=131)$ & 1.02 & $1.00-1.04$ & 0.032 \\
Neuroticism score (12-60) ( $n=131)$ & 1.06 & $0.99-1.13$ & 0.069 \\
Extraversion score (12-60) ( $n=131)$ & 0.96 & $0.88-1.04$ & 0.275 \\
Openness score (12-60) $(n=131)$ & 0.99 & $0.91-1.08$ & 0.763 \\
Agreeableness score (12-60) $(n=131)$ & 1.00 & $0.93-1.08$ & 0.970 \\
Conscientiousness score (12-60) $(n=131)$ & 0.95 & $0.87-1.03$ & 0.206 \\
\hline
\end{tabular}

Values are adjusted odds ratio and their $95 \% \mathrm{Cl}$ and $P$-values from multivariable regression model with age and gender as covariates. The results are obtained from 12 different regressions together with an apparent area under the ROC curve (AUC) of 0.78 (95\% CI: 0.65-0.91) in our sample. By obtaining the ROC curve we assess the ability of the predictors in the model to discriminate between absence and presence of $\mathrm{MOH}$. The curve is obtained by the score divided from the LASSO logistic regression and based on the included predictors.

As the sample had already been used for model selection, this estimate of model performance is overly optimistic. During the following bootstrap procedure, 141 of the 500 bootstrap samples were discarded due to convergence problems during the estimation procedure. Using the remaining bootstrap samples, we obtained an optimism estimate of 0.16 resulting in a corrected AUC of 0.62. Similarly, the corrected $95 \%$ CI for the AUC was 0.41-0.82. The AUC presents a measurement of discrimination, that is, the ability of the model to correctly classify the onset of $\mathrm{MOH}$. Given the fact that the corrected AUC is 0.62 (95\% CI: 0.41-0.82), our model (containing the predictors MIDAS score, MIDASintensity and -frequency, neuroticism score, time with moderate physical activity, educational level, hours of sleep daily and number of contacts to the headache clinic)) the model can be considered as an weak classifier for discriminating between absence and presence of $\mathrm{MOH}$.

\section{Discussion}

The main findings of the present study were that the logistic regressions indicated that the headache intensity and headache frequency were associated with onset of $\mathrm{MOH}$ and therefore could be factors to take into account to prevent the development of $\mathrm{MOH}$. This could have important implications for clinicians and highlights that the intensity and frequency of headache may help 
Table 4 Variables selected by the LASSO logistic regression

\begin{tabular}{llll}
\hline Covariates & OR (LASSO) & OR & 95\% Cl (OR) \\
\hline Sleep (hours daily) $(n=129)$ & 1.032 & 1.214 & $0.831-1.812$ \\
Moderate physical activity (hours/week) $(n=129)$ & 0.983 & 0.906 & $0.710-1.077$ \\
MIDAS score $(n=129)$ & 1.002 & 1.002 & $0.990-1.014$ \\
MIDAS-intensity (NRS 0-10) ( $n=129)$ & 1.006 & 1.011 & $0.987-1.034$ \\
MIDAS-frequency (days last 3 months) $(n=129)$ & 1.210 & 1.564 & $1.042-2.517$ \\
Neuroticism score (12-60) ( $n=129)$ & 1.017 & 1.052 & $0.981-1.133$ \\
Educational level ( $n=129)$ & 0.794 & 0.586 & $0.233-1.396$ \\
Contacts headache clinic $(n=129)$ & 1.010 & 1.068 & $0.916-1.237$ \\
\hline
\end{tabular}

The table shows estimated (shrunken) odds ratio for the selected variables. These are complemented by odds ratios and their $95 \% \mathrm{Cl}$ afterwards obtained from an ordinary multivariable logistic regression model using the same variables. Odds ratios correspond to the variables' original scale

identify the sub group at risk of developing $\mathrm{MOH}$. For this study, MIDAS questionnaire was chosen as an instrument, however other instruments measuring intensity and frequency (i.e. headache diary) could have been applicable. Furthermore, when comparing migraine patients $(-\mathrm{MOH})$ and migraine patients $(+\mathrm{MOH})$ , patients developing $\mathrm{MOH}$ reported higher intensity and frequency of headache using the MIDAS questionnaire as compared to the rest of the included migraine patients. This finding is consistent with a review that showed high headache frequency to be an important modifiable risk factor in migraine chronification progression [44] and with previous studies stating that headache frequency in particular may be a risk factor for onset $\mathrm{MOH}[8,45]$. Martelletti shows that $\mathrm{MOH}$ must be considered as sequela of chronic migraine and in light of that, it is beneficial to focus on how to reduce headache frequency among migraine patients to avoid $\mathrm{MOH}$ as a consequence [46].

Bigal et al. [47] investigated psychological profiles including the role of personality characteristics in headache chronification and observed that episodic headache patients undergoing chronification had a different personality profile compared to patients with episodic headaches [47]. In contrast, when we compared personality characteristics between migraine $(-\mathrm{MOH})$ and migraine $(+\mathrm{MOH})$ patients, we were unable to detect any differences between the groups. This can be caused by the fact that all patients at starting point were migraine patients without $\mathrm{MOH}$, and the two groups therefore remain very similar in personality characteristics, unaffected by the chronification process related to developing $\mathrm{MOH}$.

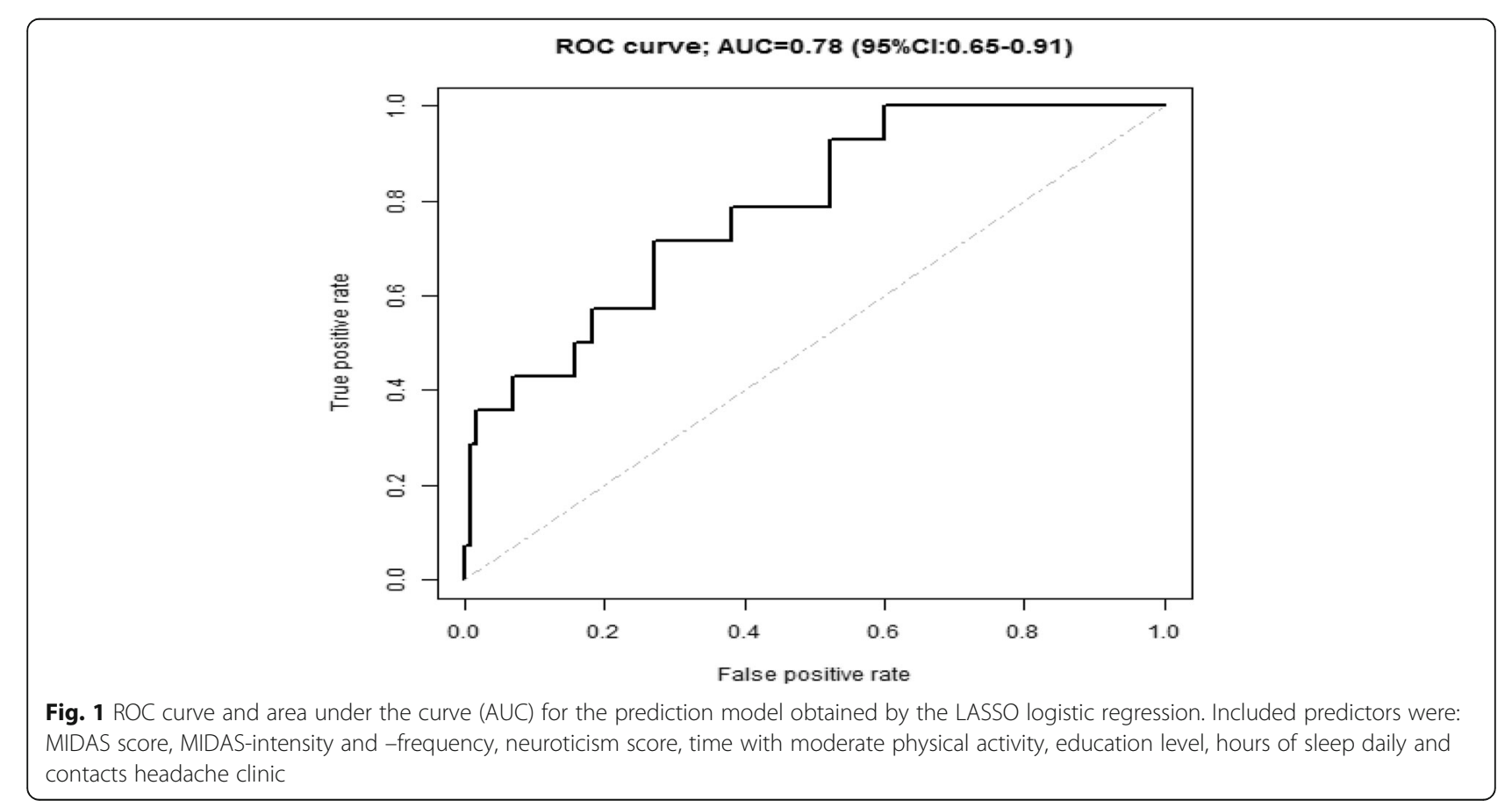


Generally, few studies have investigated associations between different headache types and personality, and the results have been ambiguous [48]. Furthermore, different measurements of personality make studies difficult to compare. For this study we chose the NEO-FFI-3 which describes personality as five domains with 12 items each without the underlying in-depth facet score [31]. This facet scores could potentially increase the sensitivity of the personality evaluations, making it possible to detect subtle differences that our method could not. This could explain the insignificant differences between $(+\mathrm{MOH})$ and $(-\mathrm{MOH})$ in the current study.

$\mathrm{MOH}$ patients tend to be more physically inactive as compared to migraine patients [8]. According to Westergaard et al. the association between $\mathrm{MOH}$ and inactivity might be due to the fact that $\mathrm{MOH}$ patients have developed an inactive lifestyle in order to avoid triggering migraine attacks [29]. In this study, both patients with migraine $(-\mathrm{MOH})$ and migraine $(+\mathrm{MOH})$ spent very few hours on physical activities per week, which probably could be caused by headache burden. The difficulty of performing physical activity among migraine patients in association to development of $\mathrm{MOH}$ seems to be irrelevant as the odds for $\mathrm{MOH}$ only decreased by $1.7 \%(\mathrm{OR}=0.983)$ for each hour spent on moderate physical activity. However, a study on physical activity and migraine treatment found that regular physical activity has beneficial effects on headache intensity and frequency, duration of headache attacks and patients well-being [49]. When $\mathrm{MOH}$ patients are physically inactive there could be a risk of maintaining $\mathrm{MOH}$ in an inappropriate circular process with inactivity, worsening in headache and increased medical intake.

This study is the first to investigate the predictive performance of models based on personality, disability and physical activity in predicting onset of $\mathrm{MOH}$. It is challenging, but clinically relevant, to identify patients at risk of developing $\mathrm{MOH}$ and therefore studies developing predictive models of headache chronification are important [50]. In our prediction model we could not determine a strong causality between the included factors of personality, disability and physical activity level in the onset of $\mathrm{MOH}$, however, we are not able to reject it either. Our findings substantiates that multiple factors potentially contributes to the onset of $\mathrm{MOH}$, which is in line with another similar predictive study [20] were they found an AUC of 0.76 when including factors of personality, gene polymorphisms, headache characteristics and lifestyle. In both studies the AUC showed a weak model classifier for the onset of $\mathrm{MOH}$.

As strength in this study the questionnaires used were not time consuming, which must be considered as an advantage, because it is easy to adopt in clinical practice. As a limitation we must consider the inclusion process since we only included patients two times per week. Therefore we are fully aware that this study represents only a sample of a larger migraine patient flow in the clinic. However this study indicates how many migraine patients developed $\mathrm{MOH}$ during treatment at a headache clinic. Further we are limited by investigating patients who were seen and treated regularly and therefore, we are unable to assess how many patients would have developed $\mathrm{MOH}$ if they had no treatment options, and if this could have caused other predictive factors to emerge. Migraineurs with particularly high medication intake are at increased risk of developing $\mathrm{MOH}$ [51]. However, detailed knowledge about prophylactic and acute medical treatment is not part of the current study, since the patients' medical ordinations changed throughout the study. Overall, only $12 \%$ of the patients developed $\mathrm{MOH}$ during the study period and the small number of patients developing $\mathrm{MOH}$ is a limitation for immediate generalization of the results. This limits the power of the study and increases the risk for errors in both the estimated size as well as direction of effects. However, one way to handle these limitations is by looking at Gelman \& Calin [52] who recommend a design calculation that provides a perspective on erroneous findings in small studies. The design calculation estimates the type $S$ error, meaning the probability of an estimate being in the wrong direction, and type $\mathrm{M}$ errors being by which factor the magnitude of an effect is overestimated. Even though the dataset was small, we succeeded on characterizing the patients developing $\mathrm{MOH}$ and modeling our data via solid statistical methods highlighting important methods for assessing easy accessible clinical data in the prevention of $\mathrm{MOH}$. The predictors included in the presented prediction model were those which best predicted presence or absence of $\mathrm{MOH}$. Due to the small effective sample size of 16 cases of $\mathrm{MOH}$ in the dataset, the complexity of resulting models was a priori limited as larger models tend to be more prone to overfitting. Further, for penalized regression methods such as LASSO logistic regression, the number of selected predictors depends crucially on the chosen strength of the penalty. In this study, its choice was governed by cross-validation. Taking the variability incorporated in this procedure into account, a wider range of other prediction models including more or less predictors becomes plausible, see also Pfeiffer \& Raymond [53] who estimated the rate of falsely included/excluded variables when applying LASSO logistic regression to a simulated dataset.

\section{Conclusion}

This study showed that the intensity and frequency of headache were associated with $\mathrm{MOH}$ onset, while there were no associations between personality and physical activity level and $\mathrm{MOH}$ onset, respectively. Our findings support that focus on headache frequency and intensity 
is essential for targeting a subgroup of migraine patients at risk of developing $\mathrm{MOH}$.

The identification of predictors of $\mathrm{MOH}$ may have important clinical implications - specifically in relation to early detection of patients at risk of developing $\mathrm{MOH}$ and in the documentation of appropriate instruments for this detection.

\begin{abstract}
Abbreviations
AUC: Area under the ROC curve is a measurement of discrimination, that is, the ability of the model to correctly classify the onset of medication-overuse headache; Cl: Confidence Interval; LASSO: Least absolute shrinkage and selection operator; MIDAS: Migraine Disability Assessment Questionnaire; MOH: Medication-overuse headache defined according to the diagnostic criteria from ICHD-III beta; NEO-FFI-3: NEO Five-Factor Inventory questionnaire; NEO-PI-R: The revised NEO Personality Inventory is the longer version of NEO-FFI-3 containing 240 items.; OR: Odds Ratio; PAS 2.1: The Physical Activity Scale, which is a questionnaire.; ROC Curve: Receiver operating characteristic curve; TTH: Tension-type headache
\end{abstract}

\section{Acknowledgements}

The authors would like to thank the Neurology Department at Hospital Southwest Jutland for the collaboration and the staff of The Headache Clinic at the Hospital Southwest Jutland, Denmark for their support with inclusion, and of course all the participating patients.

\section{Funding}

Financial support with grants from TrygFonden, Carola Jørgensen Foundation and the Danish patient organization Migræne og Hovedpineforeningen.

\section{Availability of data and materials}

Data will be made available on request.

\section{Authors' contributions}

LSM and BG conceived the idea for this article. LSM was project leader. BD and LSM performed the data analyses and data interpretations. LSM, BG, SSP, $\mathrm{BD}$ and RJ contributed with inputs to this article. The article was drafted by LSM and all authors reviewed and approved the final manuscript.

\section{Ethics approval and consent to participate}

This study was part of a larger study that was approved by the Regional Committees on Health Research Ethics for Southern Denmark (ID S20140114). Permission was obtained from the Danish Data Protection Agency (2008-58-0035).

\section{Competing interests}

The authors declare that they have no competing interests.

\section{Publisher's Note}

Springer Nature remains neutral with regard to jurisdictional claims in published maps and institutional affiliations.

\footnotetext{
Author details

${ }^{1}$ Department of Neurology, Hospital Southwest Jutland, Esbjerg, Denmark. ${ }^{2}$ The Research Unit of Health Science, Hospital of Southwest Jutland, Esbjerg and Department of Regional Health Research, University of Southern Denmark, Odense, Denmark. ${ }^{3}$ Department of Psychology, University of Southern Denmark, Odense, Denmark. ${ }^{4}$ Department of Cardiology, Odense University Hospital, Odense, Denmark. ${ }^{5}$ Epidemiology, Biostatistics and Biodemography Department of Public Health, University of Southern Denmark, Odense, Denmark. ${ }^{6}$ Danish Headache Centre, Department of Neurology, Rigshospitalet-Glostrup, University of Copenhagen, Copenhagen, Denmark.
}

Received: 23 February 2018 Accepted: 6 May 2018

Published online: 25 May 2018

\section{References}

1. Stovner LJ, Andree C (2010) Prevalence of headache in Europe: a review for the Eurolight project. J Headache Pain 11:289-299. https:// doi.org/10.1007/s10194-010-0217-0

2. Bigal ME, Lipton RB (2008) Excessive acute migraine medication use and migraine progression. Neurology 71:1821-1828. https://doi.org/10. 1212/01.wnl.0000335946.53860.1d

3. Bigal ME, Serrano D, Buse D, Scher A, Stewart WF, Lipton RB (2008) Acute migraine medications and evolution from episodic to chronic migraine: a longitudinal population-based study. Headache 48:11571168. https://doi.org/10.1111/j.1526-4610.2008.01217.x

4. Evers S, Marziniak M (2010) Clinical features, pathophysiology, and treatment of medication-overuse headache. Lancet Neurol 9:391-401. https://doi.org/10.1016/s1474-4422(10)70008-9

5. Saper JR, Da Silva AN (2013) Medication overuse headache: history, features, prevention and management strategies. CNS drugs 27:867-877. https://doi. org/10.1007/s40263-013-0081-y

6. Headache Classification Committee of the International Headache Society (IHS) (2013) The International Classification of Headache Disorders, 3rd edition (beta version). Cephalalgia 33:629-808

7. Diener HC, Limmroth V (2004) Medication-overuse headache: a worldwide problem. Lancet Neurol 3:475-483. https://doi.org/10.1016/s1474-4422(04)00824-5

8. Hagen K, Linde M, Steiner TJ, Stovner $\sqcup$, Zwart JA (2012) Risk factors for medication-overuse headache: an 11-year follow-up study. The Nord-Trondelag Health Studies. Pain 153:56-61. https://doi.org/10.1016/j.pain.2011.08.018

9. Serafini $\mathrm{G}$ et al (2012) Gene variants with suicidal risk in a sample of subjects with chronic migraine and affective temperamental dysregulation. Eur Rev Med Pharmacol Sci 16:1389-1398

10. Davis RE, Smitherman TA, Baskin SM (2013) Personality traits, personality disorders, and migraine: a review. Neurol Sci 34(Suppl 1):S7-S10. https:// doi.org/10.1007/s10072-013-1379-8

11. Silberstein SD, Lipton RB, Breslau N (1995) Migraine: association with personality characteristics and psychopathology. Cephalalgia 15:358-369; discussion 336. https://doi.org/10.1046/j.1468-2982.1995.1505358.x

12. Costa PT Jr, McCrae RR (1992) NEO PI-R:professional manual. Psychological Assesment Resources, Inc, Odessa

13. Costa PT Jr, McCrae RR (1995) Domains and facets: hierarchical personality assessment using the revised NEO personality inventory. J Pers Assess 64: 21-50. https://doi.org/10.1207/s15327752jpa6401_2

14. Mccrae RR, John OP (1992) An introduction to the five-factor model and its applications. J Pers 60:175-215

15. Lampl C et al (2016) Headache, depression and anxiety: associations in the Eurolight project. J Headache Pain 17:59. https://doi.org/10.1186/s10194-016-0649-2

16. Svedberg P, Bardage C, Sandin S, Pedersen NL (2006) A prospective study of health, life-style and psychosocial predictors of self-rated health. Eur J Epidemiol 21:767-776. https://doi.org/10.1007/s10654-006-9064-3

17. Cao M, Zhang S, Wang K, Wang Y, Wang W (2002) Personality traits in migraine and tension-type headaches: a five-factor model study. Psychopathology 35:254-258 63829

18. Merikangas KR, Stevens DE, Angst J (1993) Headache and personality: results of a community sample of young adults. J Psychiatr Res 27:187-196

19. Mongini F, Ibertis F, Barbalonga E, Raviola F (2000) MMPI-2 profiles in chronic daily headache and their relationship to anxiety levels and accompanying symptoms. Headache 40:466-472

20. Onaya T, Ishii M, Katoh H, Shimizu S, Kasai H, Kawamura M, Kiuchi Y (2013) Predictive index for the onset of medication overuse headache in migraine patients. Neurol Sci 34:85-92. https://doi.org/10.1007/s10072-012-0955-7

21. Lanteri-Minet M, Duru G, Mudge M, Cottrell S (2011) Quality of life impairment, disability and economic burden associated with chronic daily headache, focusing on chronic migraine with or without medication overuse: a systematic review. Cephalalgia 31:837-850. https://doi.org/10.1177/0333102411398400

22. Stovner $L$ et al (2007) The global burden of headache: a documentation of headache prevalence and disability worldwide. Cephalalgia 27:193-210. https://doi.org/10.1111/j.1468-2982.2007.01288.x

23. Bendtsen L et al (2014) Disability, anxiety and depression associated with medication-overuse headache can be considerably reduced by detoxification and prophylactic treatment. Results from a multicentre, 
multinational study (COMOESTAS project). Cephalalgia 34:426-433. https://doi.org/10.1177/0333102413515338

24. Wallasch TM, Kropp P (2012) Multidisciplinary integrated headache care: a prospective 12-month follow-up observational study. J Headache Pain 13: 521-529. https://doi.org/10.1007/s10194-012-0469-y

25. Steiner TJ, Stovner LJ, Vos T, Jensen R, Katsarava Z (2018) Migraine is first cause of disability in under 50s: will health politicians now take notice? J Headache Pain 19:17. https://doi.org/10.1186/s10194-018-0846-2

26. Vos T, Abajobir A, Abbafati C, Abbas K, Abate K, Abd-Allah F et al (2017) Global, regional, and national incidence, prevalence, and years lived with disability for 328 diseases and injuries for 195 countries, 1990-2016: a systematic analysis for the Global Burden of Disease Study 2016. Lancet 390: 1211-1259. https://doi.org/10.1016/s0140-6736(17)32154-2

27. Andrasik F, Grazzi L, Usai S, Kass S, Bussone G (2010) Disability in chronic migraine with medication overuse: treatment effects through 5 years. Cephalalgia 30:610-614. https://doi.org/10.1111/j.1468-2982.2009.01932.x

28. Rhodes RE, Smith NE (2006) Personality correlates of physical activity: a review and meta-analysis. Br J Sports Med 40:958-965. https://doi.org/10. 1136/bjsm.2006.028860

29. Westergaard ML, Glumer C, Hansen EH, Jensen RH (2016) Medication overuse, healthy lifestyle behaviour and stress in chronic headache: Results from a population-based representative survey. Cephalalgia 36:15-28. https://doi.org/10.1177/0333102415578430

30. Costa PT, Jr., McCrae RR (eds) (2014) NEO-PI-3 vejledning klinisk. Hogrefe Psykologiske Forlag Frederiksberg Bogtrykkeri A/S

31. McCrae RR, Costa PT (2007) Brief Versions of the NEO-PI-3. J Individ Differ 28:116-128

32. Stewart WF, Lipton RB, Dowson AJ, Sawyer J (2001) Development and testing of the Migraine Disability Assessment (MIDAS) Questionnaire to assess headache-related disability. Neurology 56:S20-S28

33. Stewart WF, Lipton RB, Kolodner K, Liberman J, Sawyer J (1999a) Reliability of the migraine disability assessment score in a population-based sample of headache sufferers. Cephalalgia 19:107-114 discussion 174

34. Stewart WF, Lipton RB, Whyte J, Dowson A, Kolodner K, Liberman JN, Sawyer J (1999b) An international study to assess reliability of the Migraine Disability Assessment (MIDAS) score. Neurology 53:988-994

35. Andersen LG, Groenvold M, Jorgensen T, Aadahl M (2010) Construct validity of a revised Physical Activity Scale and testing by cognitive interviewing. Scan J Pub Health 38:707-714. https://doi.org/10.1177/1403494810380099

36. Costa PT Jr, McCrae RR (2009) The Five-Factor Model and the NEO Inventories. In: Butcher JN, editor. Oxford library of psychology. Oxford handbook of personality assessment (pp. 299-322). New York: Oxford University Press. https://doi.org/10.1093/oxfordhb/9780195366877.013.0016

37. Jensen R, Bendtsen L (2008) Medication overuse headache in Scandinavia. Cephalalgia 28:1237-1239. https://doi.org/10.1111/j.1468-2982.2008.01742.x

38. Westergaard ML, Hansen EH, Glumer C, Jensen RH (2015) Prescription pain medications and chronic headache in Denmark: implications for preventing medication overuse. Eur J Clin Pharmacol 71:851-860. https://doi.org/10.1007/s00228-015-1858-3

39. Steyerberg EW (2009) Clinical Prediction Models. Springer, New York

40. Frieman J, Hastie T, Tibshirani R (2010) Regularization Paths for Generalized Linear Models. J Stat Softw 33:1-22

41. Sing T, Sander O, Beerenwinkel N, Lengauer T (2005) ROCR: visualizing classifier performance in R. 21:Bioinformatics, 7881

42. Robin X, Turck N, Hainard A, Tiberti N, Lisacek F, Sanchez J-C, Müller M (2011) pROC: an open-source package for R and S+ to analyze and compare ROC curves. BMC Bioinformatics 12:77

43. Kuhn M (2008) Building Predictive Models in R Using the caret Package Journal of Statistical Software 28

44. Bigal ME, Lipton RB (2006) Modifiable risk factors for migraine progression. Headache 46:1334-1343. https://doi.org/10.1111/j.1526-4610.2006.00577.x

45. Katsarava $Z$ et al (2004) Incidence and predictors for chronicity of headache in patients with episodic migraine. Neurology 62:788-790

46. Martelletti P (2018) The journey from genetic predisposition to medication overuse headache to its acquisition as sequela of chronic migraine. J Headache Pain 19:2. https://doi.org/10.1186/s10194-017-0830-2

47. Bigal ME, Sheftell FD, Rapoport AM, Tepper SJ, Weeks R, Baskin SM (2003) MMPI personality profiles in patients with primary chronic daily headache: a casecontrol study. Neurol Sci 24:103-110. https:/doi.org/10.1007/s10072-003-0094-2
48. Luconi R, Bartolini M, Taffi R, Vignini A, Mazzanti L, Provinciali L, Silvestrini M (2007) Prognostic significance of personality profiles in patients with chronic migraine. Headache 47:1118-1124. https:/doi.org/10.1111/j.1526-4610.2007.00807x

49. Narin SO, Pinar L, Erbas D, Ozturk V, Idiman F (2003) The effects of exercise and exercise-related changes in blood nitric oxide level on migraine headache. Clin Rehabil 17:624-630. https://doi.org/10.1191/0269215503cr657oa

50. Katsarava Z, Jensen R (2007) Medication-overuse headache: where are we now? Curr Opin Neurol 20:326-330. https://doi.org/10.1097/WCO.0b013e328136c21c

51. Fritsche G, Frettloh J, Huppe M, Dlugaj M, Matatko N, Gaul C, Diener HC (2010) Prevention of medication overuse in patients with migraine. Pain 151:404-413. https://doi.org/10.1016/j.pain.2010.07.032

52. Gelman A, Carlin J (2014) Beyond Power Calculations: Assessing Type S (Sign) and Type M (Magnitude) Errors. Perspect Psychol Sci 9:641-651. https://doi.org/10.1177/1745691614551642

53. Pfeiffer RM, Redd A, Raymond CJ (2017) On the impact of model selection on predictor identification and parameter inference. Comput Stat 32:667-690

\section{Submit your manuscript to a SpringerOpen ${ }^{\circ}$ journal and benefit from:}

- Convenient online submission

- Rigorous peer review

- Open access: articles freely available online

- High visibility within the field

- Retaining the copyright to your article

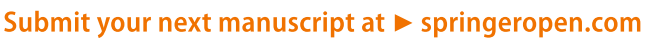

